\title{
$\mathfrak{I} \mathfrak{i} \mathfrak{a} \mathfrak{l} \mathfrak{t}$
}

Erftes \$eft.

Moberne Moraliften. I. (IVdolf Raffon.) ........... Seite 1

Die Ratbolijirung Englands. (Rubolf Bubbenfieg:) . . . . . . . . . - 27

\$bjen's nenere Dramen. (Dtto Warnad̃.) . . . . . . . . . - 55

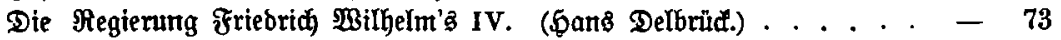

Bur (Befdidite bes Temuler $=$ Droens. (Bruno (Bebharbt.). . . . . . - 89

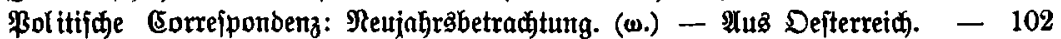

Stotizen: Dr. B. PRüller:Walbe, Eeonardo ba $\mathfrak{B t n c i . ~ - ~ R . ~ ( E g g e r s , ~ \Re a u d ~}$

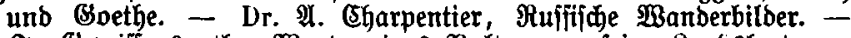

Sn Bewiffensinoth. SWorte eines $\mathfrak{B}$ alten an feine Ranbşleute. -

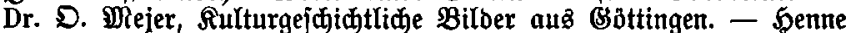

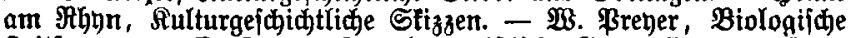

Beitfragen. - D. Säger, $\mathfrak{D a s}$ humaniftifdje Bnmmafium. - \$. v.

Broizem, Eine Shlaht ber Bufunft. . . . . . . . .

\section{3weites §eft.}

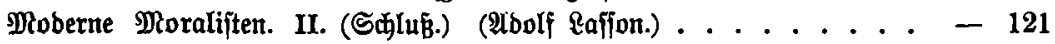

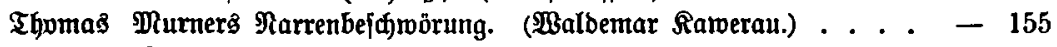

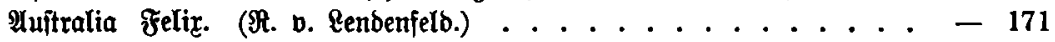

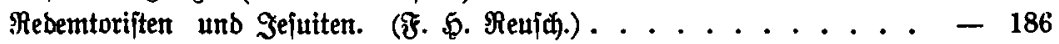

Bur Beurtheilung Dalbergsి. (Dtto Sarnat.). . . . . . . . . - 219

\$ollitijace Eorrefponbenz: Englanb. - Samoabertrag. - Frantreid). -

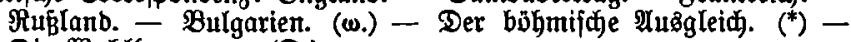

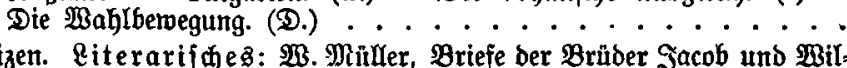

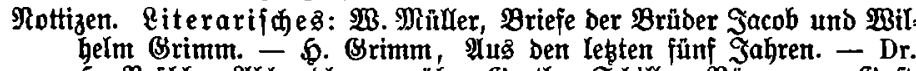
5. Próhle, $\mathfrak{A b h a n d l u n g e n ~ u ̈ b e r ~ B o e t h e , ~ S d i l l e r , ~ B u ̈ r g e r . ~ - ~ B u f t . ~}$ Sarpeles, (Boethe in \$olen. - E. (Srifebad, Bedidte von (\$. A.

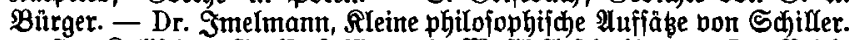
- Dr. Salijher, (S). E. Eefiting als Miujt: Alefthetifer. - Dr. Steid, (Srillparzer's Minnitphilujuphie. - R. Sepler, Das Wejen ber ßoejie. (D. (5)

Drittes \$eft.

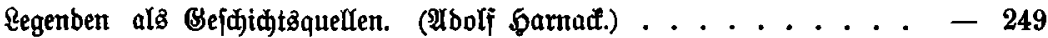

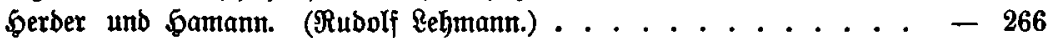

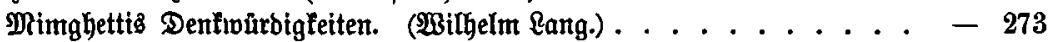

Suarez, ber Shöpfer bes preupifichen Ranbrechts und ber (Entrourf eines

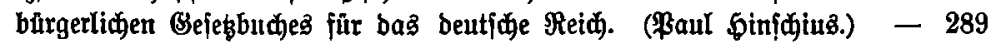

Rembrandt, als Erzieher von einem Deutjden. (WF. Bobe.) ..... - 301

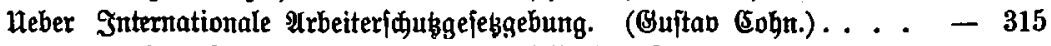

\$oltitifide (Eorreiponbenz: Die Jebruarerlaffe bea Saijerō. (w.) - Die

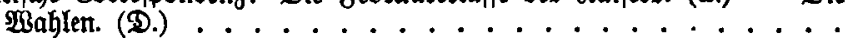

Rotizen. Siterarifळes. Neue Dramen: Gerbart ફ̧auptmann, Bor Son= menaufgang. - $\mathfrak{A}$. Solz und 3. Shlaf, Die Familie Seelide. (E. v. Silbenbruw, Der Beneralfelboberft. - E. B. Bruno, \$injel unb Sutte. - \$. SBulthaupt, Der verlorene Sohn. - \$. Suber:

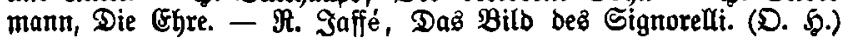




\section{Biertes \$eft.}

Die Darftellung des Sgeiligen in Der Aumit. (Earl Dlezer.). . . . . . Seite 361

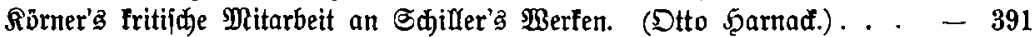

Berfaffulig, Selbitberwaltung unb Sozialreform. (Eonrad Bornhaf.) . . -410

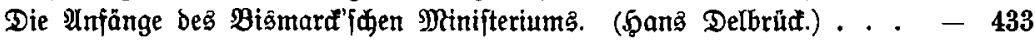

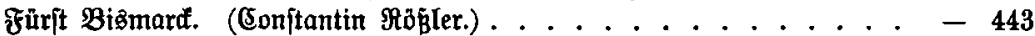

\$olitifde (Eorrefpondenz: Der Ranzlerivedjel. (D.). . . . . . . . - 464

Rotizen unb Befprechungen: Iman Rermolieff. (艿. Rovpmamt.) - Qi te= rarijaes: Frtebrid Dernburg, Der Dherftolze. - 5. Tonote, Int

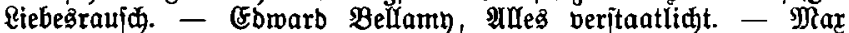
Fobredt, Reue Ropellen. - $\mathfrak{A}$. Stern, Die lekten Symanititen. --

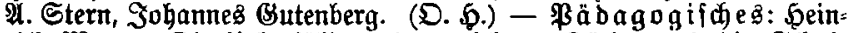

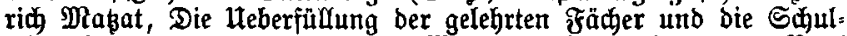

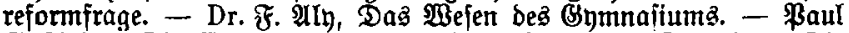
(Bübfelot, Die Ergiehung ber beutiden Sugent. (D. 5.) - Die

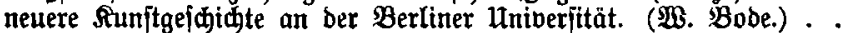

\section{ช̛unftes ఏeft.}

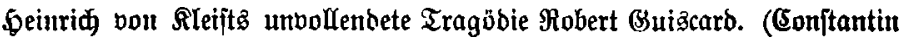
$\Re$ $\Re_{\mathrm{\beta}}$ ler.) . . . . . . . . . . . . . . . -485

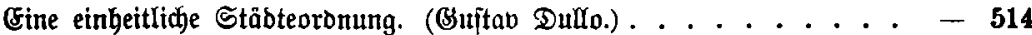

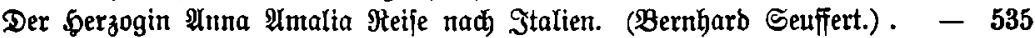

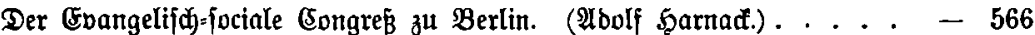

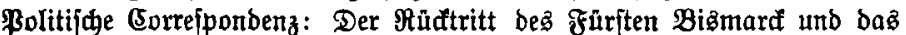

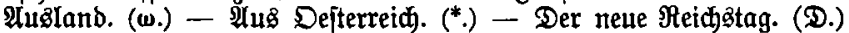

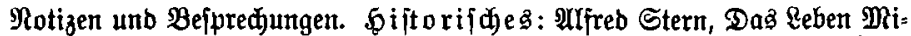
rabeaus. - Emil Daniels, \&ubrig X VI. und Marie Ântoinette auj ber Fludt. - \&ady $\mathfrak{B l e n n e r h a j f e t , ~ F r a u ~ v o n ~ S t a e ̈ l . ~ ( D . ) ~ - ~} \mathfrak{R}$ ational of onomifdes: DUrr. S. Conrab, R. (Fliter, $\mathfrak{B}$. Reris, Edg. Roening,

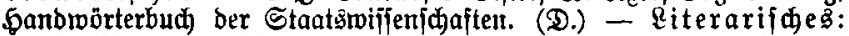

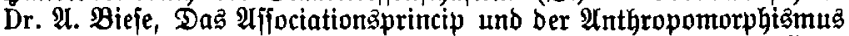
in Der Alefthetif. - D. Trautmann, Rehre vom Sdünen. - Emil Reid, Bian Bincenzo Brabina alā Aejthetifer. - E. Â. $\mathfrak{B a y e r , ~}$

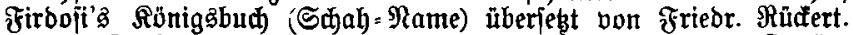

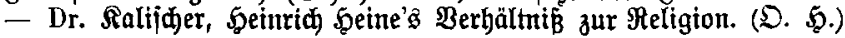

\section{Sechjtes Seft.}

Der heutige Stand ber Feftungg̊frage. (v. B.). . . . . . . . . - 601

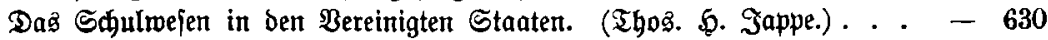

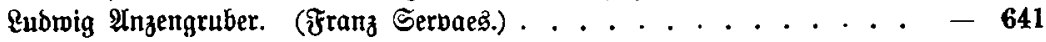

Die mirthjळaftlidje ßeripeftive Der gegenwärtigen Rohnbewegung. (Dr. T. Babifer.) . . . . . . . . . . . . . .

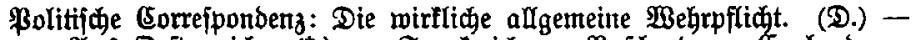

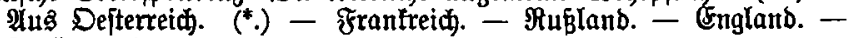

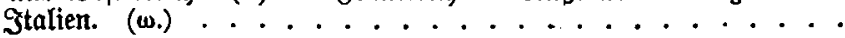

Rotizen unb Bejpredungen. Shiller: S. Dinor, Shaller. Sein Reben unb feine $\mathfrak{B e r f e . ~ - ~ \& . ~ B e l l e r m a n n , ~ S d j i l l e r ' s ~ D r a m e n . ~ B e i t r a ̈ g e ~}$

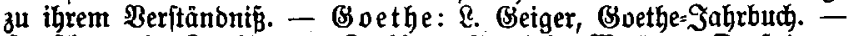

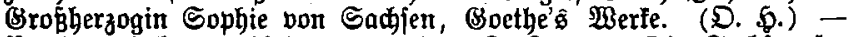
Rationalofonomifdes. Dr. jur. R. Rärger, Die Sadjengän= gerei. (D.) . . . . . . . . . . . . . 